\title{
miRNA-22 suppresses colon cancer cell migration and invasion by inhibiting the expression of T-cell lymphoma invasion and metastasis 1 and matrix metalloproteinases 2 and 9
}

\author{
BO LI ${ }^{1}$, YAN SONG ${ }^{1}$, TONG-JUN LIU ${ }^{1}$, YOU-BIN CUI ${ }^{2}$, YANG JIANG ${ }^{1}$, ZHONG-SHI XIE $^{1}$ and SHU-LI XIE $^{3}$ \\ ${ }^{1}$ Department of Colorectal and Anal Surgery, The Third Affiliated Hospital of Jilin University; \\ Departments of ${ }^{2}$ Thoracic Surgery and ${ }^{3}$ Hepatobiliary Surgery, The First Affiliated \\ Hospital of Jilin University, Changchun, P.R. China
}

Received December 11, 2012; Accepted February 3, 2013

DOI: $10.3892 /$ or.2013.2300

\begin{abstract}
Emerging evidence has demonstrated the altered expression of mRNAs in cancer development and progression. In this study, the precise role of miRNA-22 (miR-22) in colon cancer cells was investigated. Upon transfection with a miR-22 expression vector, the viability of HCT-116 human colon cancer cells was significantly reduced and tumor cell migration and invasion capacity were also suppressed. Computational in silico analysis predicted that T-cell lymphoma invasion and metastasis 1 (TIAM1) is a target gene of miR-22. This was confirmed by qRT-PCR and western blotting, which showed that miR-22 expression inhibited TIAM1 mRNA and protein expression, respectively. In addition, the expression of proinvasive gene matrix metalloproteinases 2 and 9 (MMP-2 and MMP-9) and pro-angiogenic protein vascular endothelial growth factor (VEGF) were also reduced by miR-22 expression. Collectively, these data suggest that miR-22 may act as a tumor suppressor in colon cancer, most likely by targeting TIAM1 expression.
\end{abstract}

\section{Introduction}

Colorectal cancer is a significant health burden worldwide, accounting for over 1,2 million new cancer cases and more than 600,000 cases of cancer-related mortality annually (1). However, incidence of this type of cancer has recently decreased in several Western countries, largely due to increased awareness, early detection and prevention measures (2). In addition, advancements in treatments have improved the survival of patients with this disease. To date, surgery

Correspondence to: Dr Yan Song, Department of Colorectal and Anal Surgery, The Third Affiliated Hospital of Jilin University, 126 Xiantai Street, Changchun, Jilin 130033, P.R. China

E-mail: caifu2005@hotmail.com

Key words: miRNA-22, colon cancer, invasion, T-cell lymphoma invasion and metastasis 1 is curative for the early stages of colorectal cancer, while chemotherapy plus surgery is used to treat the later stages of the disease. However, most patients eventually develop metastatic disease and succumb to colorectal cancer. Thus, novel approaches for the early detection, treatment and suppression of tumor progression are required to effectively control this disease.

microRNAs (miRNAs) are a group of naturally-occurring, small non-coding RNAs, 18-22 nucleotides long, that regulate target gene expression by specifically inhibiting the translation of mRNA or inducing mRNA degradation (2,3). Accumulating evidence has demonstrated that miRNAs play a critical role in regulating a variety of physiological and pathological processes in the human body, such as cell differentiation, morphogenesis and tumorigenesis $(4,5)$. In human cancer, miRNAs can function either as oncogenes or tumor suppressor genes in regulating tumor development and progression (6), depending on the target gene. Thus, identifying and understanding the molecular targets of miRNAs by using computational in silico analysis with a specific algorithm, such as TargetScan (7), could elucidate the signaling cascades involved in colorectal cancer progression.

It was recently shown that miR-22 is dysregulated and functions as a tumor suppressor gene in several types of cancer. In addition, its altered expression is associated with the poor prognosis of colon cancer. Furthermore, previous studies showed that miR-22 expression inhibited the proliferation of A539 and H1299 lung cancer cells by post-transcriptional regulation of ErB3 expression (8), while in breast cancer, miR-22 inhibited estrogen receptor $\alpha$ (ER- $\alpha)$ and ectopic viral integration site-1 (EVI-1) expression to suppress tumor cell proliferation and metastatic potential (9-11). miR-22 expression also inhibited hepatocellular carcinoma cell proliferation by targeting HDAC4 (12), while inhibiting ovarian cancer cell migration and invasion by targeting T-cell lymphoma invasion and metastasis 1 (TIAM1) expression $(13,14)$. Furthermore, it is showed that TNFAIP8-rs11064 variant $G$ allele weaken the binding affinity of miR-22 to TNFAIP8 3'-UTR contributing to cervical cancer risk (38). Similar data were shown in colon cancer cells, where PTEN expression was downregulated by miR-22, which contributed to sensitizing paclitaxel-induced 
chemoresistance $(15,16)$. miR-22 expression was also shown to regulate hypoxia signaling in colon cancer cells by suppressing the expression of hypoxia inducible factor-1 $\alpha$ (HIF-1 $\alpha)(17)$, while miR-22 expression was induced by vitamin $\mathrm{D}$ treatment to have anti-proliferative and anti-migratory functions in colon cancer cells (18). These data indicate that there may be multiple genes regulated by miR-22 in different types of cancer.

In the present study, we investigated the effect of miR-22 expression on colorectal cancer cell viability, migration and invasion. In addition, computational in silico analysis with TargetScan revealed TIAM1 as a potential miR-22 target gene; we subsequently performed qRT-PCR, western blotting and immunocytochemistry to confirm this finding. TIAM1 is a guanine nucleotide exchange factor protein that modulates the activity of Rho GTP-binding protein Rac1; in turn, Rac1 regulates the reorganization of cytoskeletal structure and promotes cell adhesion and movement, thereby contributing to the invasion and metastasis of tumor cells (19). In normal tissues, with the exception of the brain and testis, TIAM1 expression is absent or at low levels. By contrast, TIAM1 is highly expressed in different types of cancer (particularly metastatic cancer), including colon (20), liver (21-23), kidney (24), esophagus (25), nasopharynx (26), ovary (13), breast (27) and lung cancer (28).

\section{Materials and methods}

Cell culture and transfection. The HCT-116 human colorectal cancer cell line was purchased from the American Type Culture Collection (Manassas, VA, USA) and routinely cultured in McCoy's 5A modified medium supplemented with $10 \%$ fetal calf serum (FCS), $100 \mathrm{U} / \mathrm{ml}$ penicillin and $100 \mu \mathrm{g} / \mathrm{ml}$ streptomycin (Invitrogen, Carlsbad, CA, USA) in a $37^{\circ} \mathrm{C}$ humidified incubator containing $5 \% \mathrm{CO}_{2}$. hsa-miR-22 precursor sequences (5'-AGUUCUUCAGUGGCAAGCUUUA-3') were cloned into the SD13 expression vector (GeneChem, Shanghai, China), amplified and sequence-confirmed prior to use. The plasmid encoding the miR-22 precursor was labeled SD13-hsa-miR-22.

To induce miR-22 expression in HCT-116 cells, we transfected SD13-hsa-miR-22 or control vector into HCT-116 cells using Lipofectamine 2000 (Invitrogen) according to the manufacturer's instructions. Briefly, cells plated at $70 \%$ confluence in 24-well plates were transfected with $0.8 \mu \mathrm{g}$ SD13-hsa-miR-22 or control plasmid and re-fed with fresh medium $4 \mathrm{~h}$ after transfection. The expression of green fluorescent protein (GFP) was monitored in cells as an indicator of transfection efficiency and total RNA was isolated $48 \mathrm{~h}$ after transfection to verify gene expression.

Online search of GenBank databases. To predict miR-22 target genes, we performed online searches of two different Genbank databases; i.e., miRNA database; http://www.sanger. ac.uk/Software/Rfam/mirna and TargetScan algorithm (http:// www.targetscan.org).

$q R T-P C R$. Total RNA was isolated using RNAiso for Small RNA (Takara, Japan), according to the manufacturer's instructions, and then reverse transcribed into cDNA using One Step PrimeScript ${ }^{\circledR}$ miRNA cDNA Synthesis kit (Perfect Real Time, Takara, Japan). The newly synthesized cDNA was then used as a template for detection of miR-22 and other genes.
Specifically, 100 ng cDNA was mixed with SYBR Premix Ex $\mathrm{Taq}^{\mathrm{TM}} \mathrm{II}$ (Perfect Real Time) in a $20-\mu 1$ reaction. All reactions were run in triplicate with the ABI PRISM ${ }^{\circledR} 7300$ Real-Time PCR system (Applied Biosystems, Foster City, CA, USA) using primers specific for miR-22, $\beta$-actin, matrix metalloproteinases 2 and 9 (MMP-2 and MMP-9), TIAM1 and vascular endothelial growth factor (VEGF) (Table I). Reaction conditions were: $50^{\circ} \mathrm{C}$ for $2 \mathrm{~min}$ and $95^{\circ} \mathrm{C}$ for $1 \mathrm{~min}$, followed by 40 cycles of $95^{\circ} \mathrm{C}$ for $5 \mathrm{sec}$ and $60^{\circ} \mathrm{C}$ for $1 \mathrm{~min}$. The relative expression of mRNA was analyzed using 7300 System SDS software. U6 small nuclear RNA was used as an internal control.

MTT cell viability assay. To determine the effect of miR-22 on colorectal cell viability, we performed the MTT assay. Briefly, HCT-116 colon cancer cells transfected with control vector (NC) or SD13-hsa-miR-22 were plated in a 96-well plate at a density of $1 \times 10^{4}$ cells per well and grown for up to 7 days in complete cell culture media. Thereafter, $20 \mu 1$ of 10X MTT reagent (250 mg of 3-[4,5-dimethylthiazol-2-yl]2,5-diphenyltetrazolium bromide; Sigma, St. Louis, MO, USA) was added and incubated with the cells for $4 \mathrm{~h}$. The media was subsequently aspirated and replaced with DMSO and the cells were incubated at room temperature for $15 \mathrm{~min}$ with vigorous shaking. Optical absorbance was measured spectrophotometrically at $490 \mathrm{~nm}$ daily for 7 days to quantify cell proliferation. Cell proliferation curves were plotted based on absorbance.

Immunocytochemistry. To detect changes in gene expression in HCT-116 cells in the absence or presence of miR-22 transfection, we performed immunocytochemistry using a streptavidin peroxidase method. The protein expression of MMP-2, MMP-9, VEGF and TIAM1 was assessed using the corresponding antibodies in colon cancer cells. Following transfection, the cells were fixed in $10 \%$ formalin for $10 \mathrm{~min}$ and then treated with $0.5 \%$ Triton X-100 for 10 min. After blocking in $20 \%$ normal serum, the cells were incubated with a primary antibody for $1 \mathrm{~h}$ and a biotinylated secondary antibody for $10 \mathrm{~min}$, followed by incubation with streptavidinconjugated horseradish peroxidase for $15 \mathrm{~min}$ at $37^{\circ} \mathrm{C}$. The immune reaction was visualized with DAB (3,3'-diaminobenzidine) and images were captured with Image-Pro Plus (Media Cybernetics, Bethesda, MD, USA).

In vitro tumor cell migration and invasion assay. To determine the effects of miR-22 on colorectal cell migration and invasion, we performed cell migration and invasion assays. In brief, Matrigel was thawed on ice overnight at $4^{\circ} \mathrm{C}$ and diluted with cold basal medium. Diluted Matrigel $(30 \mu \mathrm{l})$ was added to the upper chamber of the two-chamber Transwell system $(8-\mu$ m pore size and 6.5-mm diameter; Corning, Chelmsford St. Lowell, MA, USA) and the plate was allowed to sit at room temperature at $37^{\circ} \mathrm{C}$ for $30 \mathrm{~min}$. Thereafter, $200 \mu \mathrm{l}$ of cell suspension $\left(1 \times 10^{6}\right.$ cells $\left./ \mathrm{ml}\right)$ in McCoy's 5A medium supplemented with $0.1 \%$ FCS was plated on the top chamber of the coated transwell. The bottom chamber was filled with $600 \mu 1 \mathrm{McC}$ cy's 5A medium containing $30 \% \mathrm{FBS}$ and served as a chemo-attractant. After a 48 -h incubation at $37^{\circ} \mathrm{C}$, cells on the upper surface of the filter were carefully removed with a cotton swab. Cells that 
Table I. Primers for qPCR.

\begin{tabular}{llr}
\hline Primer & \multicolumn{1}{c}{ Sequence } & Size of PCR product (bp) \\
\hline hsa-miR-22 & Purchased from RiboBio (Guangzhou, China) & 88 \\
U6 & Purchased from RiboBio (Guangzhou, China) & 96 \\
$\beta$-actin & 5'-CTGGAACGGTGAAGGTGACA-3' & 140 \\
& 5'-AAGGGACTTCCTGTAACAACGCA-3' & \\
MMP-2 & 5'-GCTGGAGACAAATTCTGGAGATACA-3' & 281 \\
& 5'-GTATCGAAGGCAGTGGAGAGGA-3' & \\
MMP-9 & 5'-GCTGGCAGAGGAATACCTGTAC-3' & 112 \\
& 5'-CAGGGACAGTTGCTTCTGGA-3' & \\
TIAM1 & 5'-AAGACGTACTCAGGCCATGTCC-3' & 253 \\
VEGF & 5'-GACCCAAATGTCGCAGTCAG-3' & \\
& 5'-AAGATCCGCAGAC GTGTAAATGTT-3' & 101
\end{tabular}

miR-22 expression level

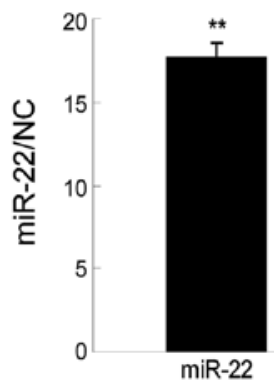

miR-22 expression level

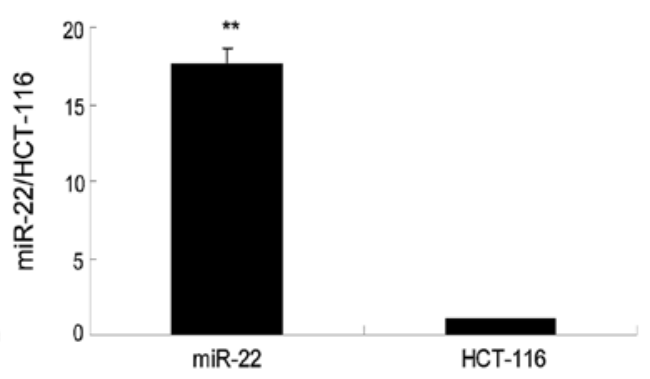

Figure 1. miR-22 expression in colon cancer cells. HCT-116 human colon cancer cells transfected with a negative control (NC) or miR-22 expression vector were subjected to qRT-PCR analysis of miR-22 mRNA expression levels.

had traversed the membrane were fixed in methanol at $4^{\circ} \mathrm{C}$ for $30 \mathrm{~min}$ and then stained with $0.1 \%$ crystal violet for $20 \mathrm{~min}$. To quantify the migrated cells microscopically, cells were counted in five random fields/filter (magnification, $x 400$ ). The migration assay was performed in a similar fashion in a transwell (Corning) without Matrigel coating.

Protein extraction and western blot analysis. To determine the effects of miR-22 on the regulation of expression of different genes, in colorectal cells, HCT-116 cells transfected with miR-22 or NC expression vectors were washed twice with cold phosphate-buffered saline (PBS), harvested by scraping and lysed in a lysis buffer (Takara). Following centrifugation, the supernatant was collected and protein concentration was determined using a Protein Assay kit II (Bio-Rad, Hercules, CA, USA). For western blotting, $50 \mathrm{mg}$ of each sample was mixed with sodium dodecyl sulfate (SDS) loading buffer, heated at $95^{\circ} \mathrm{C}$ for $5 \mathrm{~min}$, resolved on $12 \%$ SDS-polyacrylamide gel electrophoresis (SDS-PAGE) gels and transferred onto polyvinylidene difluoride (PVDF) membranes (Perkin-Elmer Life Sciences, Waltham, MA, USA). Following incubation for $1 \mathrm{~h}$ with $5 \%$ skim milk in PBS, the membranes were incubated overnight at $4{ }^{\circ} \mathrm{C}$ with primary antibodies against
MMP-2, MMP-9, VEGF and TIAM1. Protein bands of interest were visualized using enhanced chemiluminescence (ECL), followed by exposure to X-ray film following incubation with horseradish peroxidase-conjugated secondary antibodies. The film was scanned and analyzed with Gel Image System software (Simon, ProteinSimple, Santa Clara, CA, USA).

Statistical analysis. Results are expressed as the means \pm SD (standard deviation) unless otherwise specified. Statistical analyses were performed between two groups with the Student's t-test and between multiple groups by ANOVA. P-values $\leq 0.05$ were considered to indicate statistically significant differences.

\section{Results}

Expression of miR-22 in colon cancer cells. In this study, we used qRT-PCR to examine miR-22 expression in HCT-116 colorectal cancer cells and found that it was weakly expressed (Fig. 1). We therefore transfected a miR-22 expression vector into this cell line. Our data showed that the expression level of transfected miR-22 was $17.65 \pm 0.98$-fold higher than that of $\mathrm{NC}$ or parental cells (Fig. 1). 


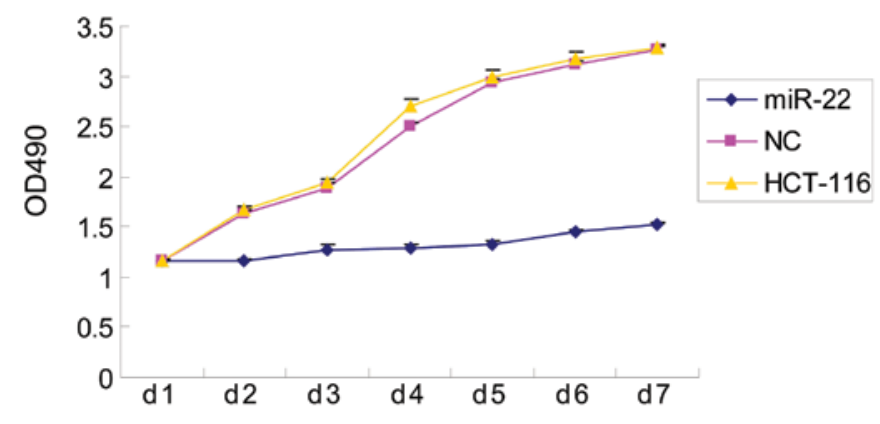

Figure 2. Effects of miR-22 expression on colon cancer cell viability. Parental, NC and miR-22-expressing HCT-116 cells were subjected to an MTT assay and optical absorbance was measured daily for up to 7 days. Tumor cell viability curves of these three groups of cells were plotted accordingly.

Effect of miR-22 on colon cancer cell proliferation. Next, we assessed the effect of miR-22 on colon cancer cell proliferation and found that miR-22 significantly inhibited cell viability (Fig. 2). Specifically, the optical absorbance rates of parental and $\mathrm{NC}$ cells were $>2$-fold higher than those of miR-22-transfected HCT-116 cells $(\mathrm{P}<0.05)$. By contrast, there was no significant difference between parental and $\mathrm{NC}$ control cells during the 7 days of culture.

Effects of miR-22 on colon cancer cell migration and invasion. Previous studies demonstrated that miR-22 was able to inhibit the invasion and metastasis of lung (8), breast (22) and ovarian cancer cells (15). This study investigated whether miR-22 is also able to suppress colon cancer cell invasion. Therefore, parental, NC and miR-22-expressing HCT-116 cells were subjected to transwell migration and invasion assays. We found that the number of cells that migrated through the pores in miR-22-expressing cells was $59 \pm 3.75$, which was significantly lower than that of $\mathrm{NC}(81 \pm 4.65)$ or parental cells $(78 \pm 3.97)(\mathrm{P}<0.05$; Fig. $3 \mathrm{~A})$, suggesting that miR-22 expression suppressed the migration of colon cancer cells. Similarly, the invasion assay showed that the number of cells that invaded the Matrigel and migrated through the pore in the membrane was markedly lower in tumor cells expressing miR-22 $(29 \pm 1.75)$ compared to parental $(42 \pm 2.65)$ or $\mathrm{NC}$ cells (38 \pm 2.32$)$ (Fig. 3B). Collectively, these results suggest that induction of miR-22 expression significantly inhibits the migration and invasion of colon cancer cells.

Effects of miR-22 on TIAM1, MMP-2, MMP-9 and VEGF expression in colon cancer cells. To elucidate the underlying molecular events responsible for miR-22-mediated inhibition of colon cancer cell migration and invasion, we first performed an in silico analysis using TargetScan to search potential miR-22 target genes and found that TIAM1, a Rho GTPase that was shown to be associated with colon cancer metastasis $(29,30)$, is a target of miR-22. We then verified our finding in miR-22-transfected HCT-116 cells. As shown in Fig. 4, levels of TIAM1 mRNA in negative control shRNA-transfected HCT-116 cells were $8.05 \pm 0.64$-fold higher than those of miR-22-transfected cells. Western blot analysis showed that TIAM1 protein expression was $>4$-fold higher in both the parental or NC cells than in miR-22-transfected cells (Fig. 5A). Immunocytochemistry confirmed our western blotting data (Fig. 5B). Collectively, these data demonstrate that miR-22 is able to suppress TIAM1 mRNA and protein expression and, consequently, to contribute to the suppression of colon cancer cell migration and invasion.

Furthermore, we analyzed expression of matrix MMP-2 and MMP-9, which play critical roles in cancer cell invasion and metastasis (31), as well as the expression of pro-angiogenic protein VEGF (32) in these cells. As shown in Figs. 4 and 5, the expression of MMP-2, MMP-9 and VEGF was
A
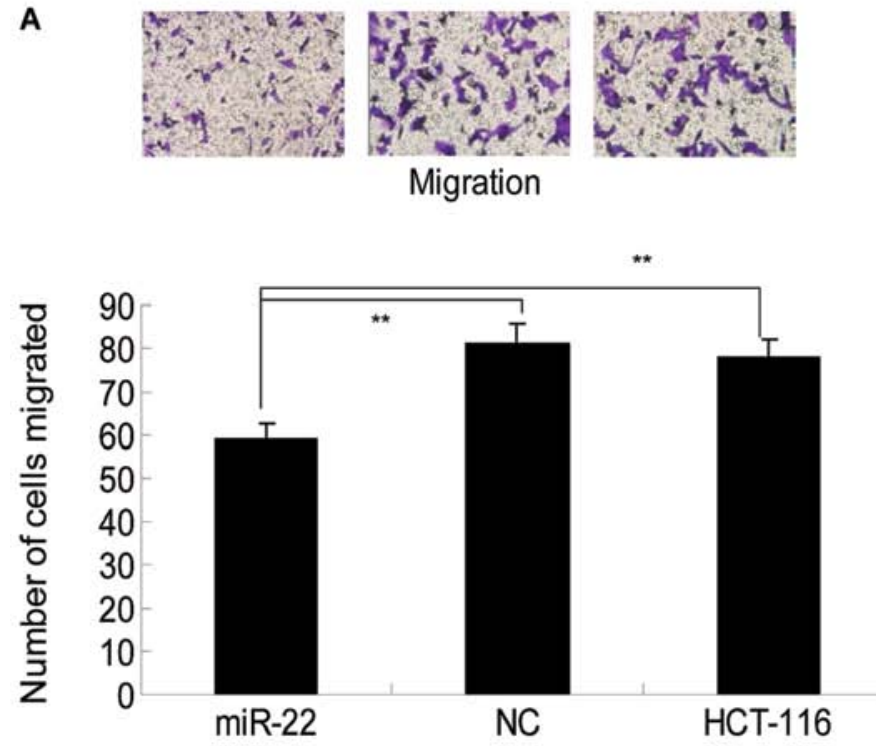

B
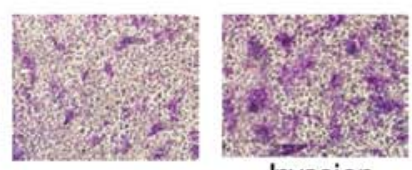

Invasion

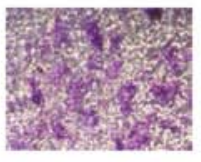

**

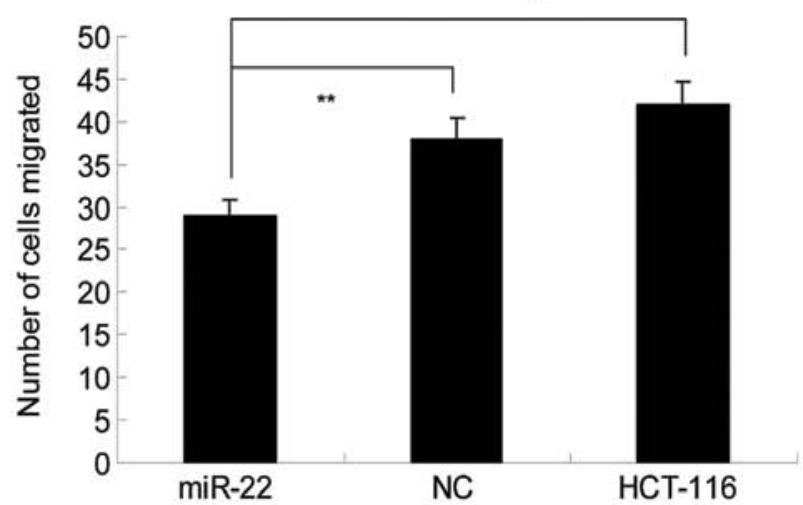

Figure 3. Effects of miR-22 transfection on suppression of colon cancer cell migration and invasion capacity. (A) Parental, NC and miR-22-expressing HCT-116 cells were subject to migration assay. (B) Parental, NC and miR-22-expressing HCT-116 cells were subject to invasion assay. Columns indicate three independent experiments; bars, $\mathrm{SD} ;{ }^{* *} \mathrm{p}<0.01$. 

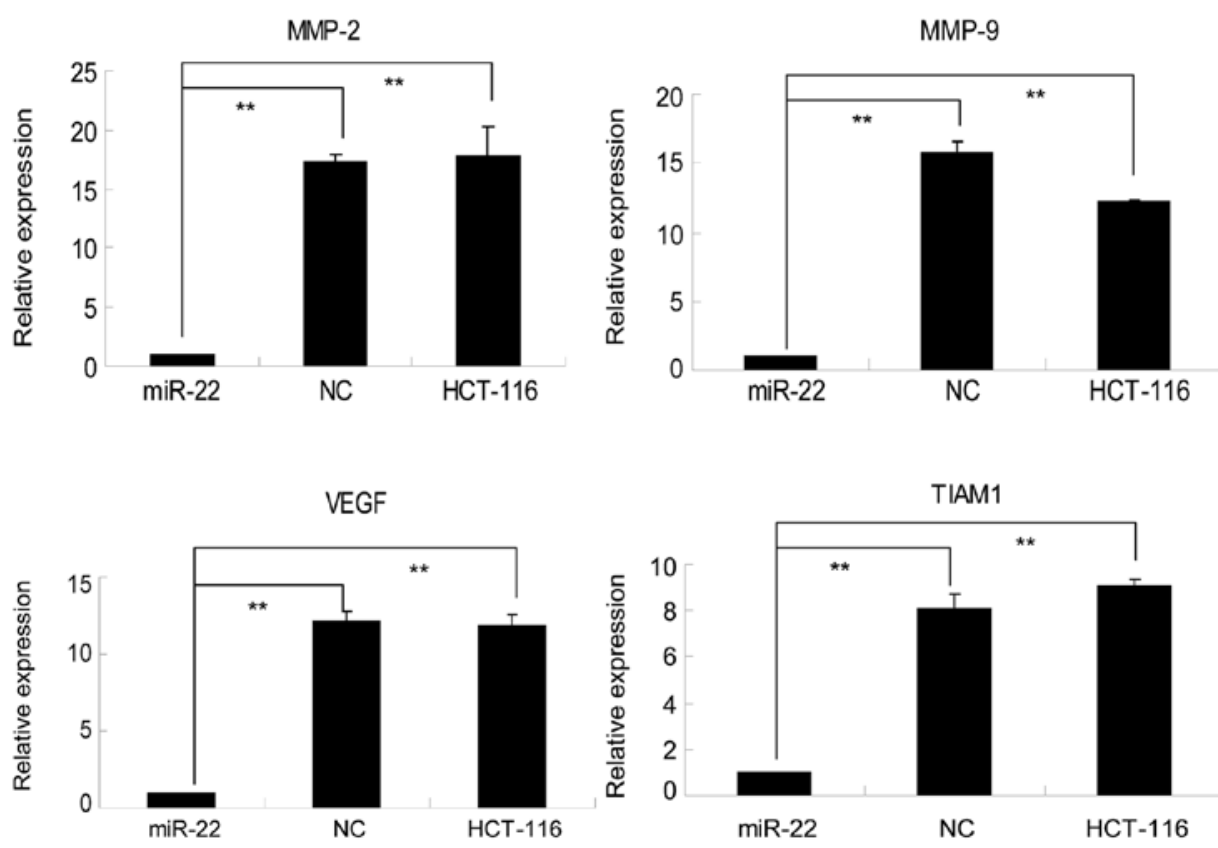

Figure 4. Effects of miR-22 transfection on regulation of TIAM1, MMP-2, MMP-9 and VEGF mRNA expression. Parental, NC and miR-22-expressing HCT-116 cells were subjected to qRT-PCR analysis of TIAM1, MMP-2, MMP-9 and VEGF expression. Columns indicate three independent experiments; bars, $\mathrm{SD} ;{ }^{* *} \mathrm{p}<0.01$

A

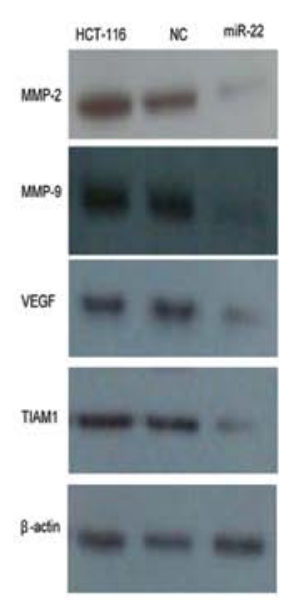

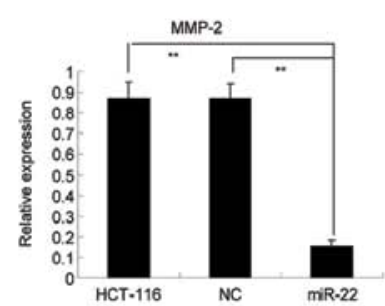
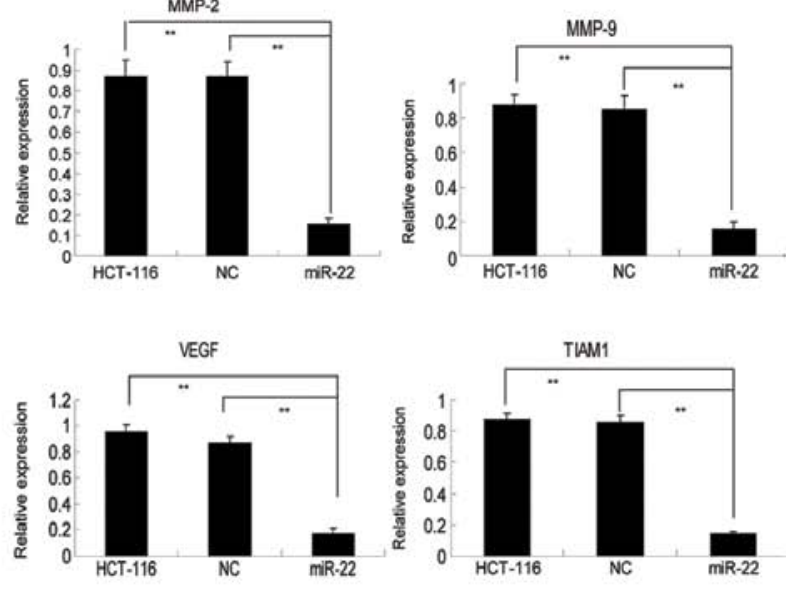

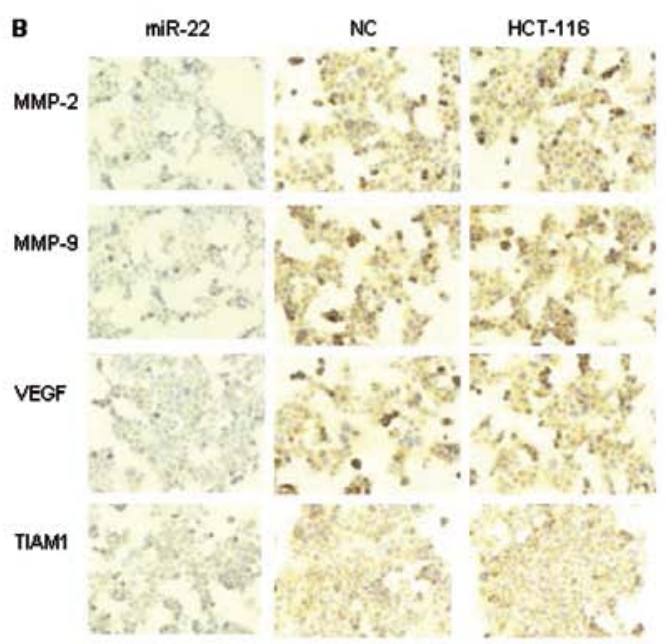

Figure 5. Effects of miR-22 transfection on the regulation of TIAM1, MMP-2, MMP-9 and VEGF protein expression. (A) Parental, NC and miR-22-expressing HCT-116 cells were subjected to western blot analysis of TIAM1, MMP-2, MMP-9 and VEGF protein expression. The intensity of bands representing proteins of interest was quantified. (B) Immunocytochemical analysis of TIAM1, MMP-2, MMP-9 and VEGF expression in parental, NC and miR-22-expressing HCT-116 cells. Columns indicate three independent experiments; bars, $\mathrm{SD} ;{ }^{* *} \mathrm{p}<0.01$.

well associated with miR-22 expression at both the transcriptional and translational levels. In particular, in parental and NC cells, the level of MMP-2 mRNA expression was $17.27 \pm 0.64$ and $17.74 \pm 2.53$-fold higher, respectively, than that in miR-22-transfected cells, while levels of MMP-9 mRNA in parental and NC cells were $15.76 \pm 0.85$ and $16.04 \pm 0.23$-fold higher, respectively, than those in miR-22-transfected cells. Similarly, VEGF expression levels in parental and NC cells were $12.14 \pm 0.61$ and $11.78 \pm 0.82$-fold higher, respectively, than those in miR-22-transfected cells (Fig. 4). A similar trend was observed at the protein level (Fig. 5).

\section{Discussion}

Emerging evidence has demonstrated that the altered expression of miR-22 plays a role in the progression of different types of cancer; however, the precise role of miR-22 in colon cancer cells remains elusive. In the present study, we overexpressed miR-22 in HCT-116 human colon cancer cells. Compared to the negative control, overexpression of miR-22 significantly inhibited the proliferation of colon cancer cells in vitro. Moreover, expression of miR-22 also exerted inhibitory effects on the suppression of HCT-116 cell migration and 
invasion. Molecularly, we evaluated and verified that miR-22 expression downregulated TIAM1 expression which, in turn, downregulated MMP-2, MMP-9 and VEGF expression in HCT-116 cells. This study indicated that the inhibitory effects of miR-22 on colorectal cancer cell viability and invasion merit further investigation as a novel strategy for the treatment of colorectal cancer and may thus be used to prevent colorectal cancer metastasis.

miR-22 has been extensively studied in different human types of cancer in vitro and ex vivo, but very few studies have reported its role in colorectal cancer. miR-22 is highly conserved across several vertebrate species. Early studies showed that miR-22 plays a role in erythrocyte maturation (33). More recent data has demonstrated that miR-22 expression is altered in various types of cancer and plays a role in tumorigenesis; thus, it is thought to function as a tumor suppressor in human cancer. It should be pointed out that the relative expression levels of miR-22 were significantly lower in colorectal cancer tissues than those in the normal adjacent mucosa (39). However, the role of miR-22 in colorectal cancer remains unknown. In the present study, we showed that overexpression of miR-22 reduced colorectal cancer cell viability and migration and invasion capacity, indicating that miR-22 may be useful in the control of colorectal cancer progression in future clinic.

Furthermore, in terms of miR-22-targeting genes, previous studies showed that miR-22 is able to target a variety of genes, such as HDAC4 (11) and c-Myc (34) and HIF-1 $\alpha$ (17). Nevertheless, in the present study, TIAM1 was predicted to be the target gene of miR-22 based on computational in silico analysis. TIAM1 has been shown to regulate migration, invasion and apoptosis of colon cancer cells and TIAM1 expression contributed to the metastatic phenotype of colon cancer in nude mice (32). This finding is in accordance with our current observation that miR-22 expression inhibited colorectal cancer cell viability and invasion, possibly via downregulation of TIAM1 expression. However, further confirmation is required to determine whether these changes that occur in colorectal HCT-116 cells upon miR-22 transfection are due to decreases in TIAM1 expression. Thus, in future studies, we will manipulate TIAM1 expression in miR-22-transfected tumor cells to demonstrate that TIAM1 mediates miR-22 effects on colon cancer cell viability and invasion.

Aside from TIAM1, the present study also showed that gene expression of MMP-2, MMP-9 and VEGF was significantly reduced upon transfection with miR-22 expression in colon cancer cells. To date, there are no studies showing the association between TIAM1 and MMP-2 and MMP-9 expression; however, a previous study demonstrated that TIAM1 promoted lymphangiogenesis by regulating Rac/COX-2/ VEGF-C signaling (35). In turn, Rac-1 promotes homophilic complex formation of MT-MMP at the lamellipodia and promotes cell migration $(36,37)$. Therefore, miR-22 might target TIAM1, which in turn leads to defective MMP-2 and MMP-9 activation and cellular invasion of colon cancer cells. Since both TIAM1 and VEGF expression were compromised upon expression of miR-22, we proposed that the inhibitory action of miR-22 on colon cancer proliferation is mediated via TIAM1, which then regulates VEGF expression. Further studies are required to confirm this hypothesis.
In conclusion, our data suggest that the pharmacological manipulation of miR-22 expression may be a tool for the diagnosis and treatment of colon cancer. However, as this study is proof-of-principle, further investigations are required to firmly establish the role of miR-22 in human colon cancer.

\section{Acknowledgements}

The authors thank Medjaden Bioscience Limited for assisting in the preparation of this manuscript.

\section{References}

1. Jemal A, Bray F, Center MM, Ferlay J, Ward E and Forman D: Global cancer statistics. CA Cancer J Clin 61: 69-90, 2011.

2. Bartel DP: MicroRNAs: target recognition and regulatory functions. Cell 136: 215-233, 2009.

3. Filipowicz W, Bhattacharyya SN and Sonenberg N: Mechanisms of post-transcriptional regulation by microRNAs: are the answers in sight? Nature reviews. Genetics 9: 102-114, 2008.

4. Esquela-Kerscher A and Slack FJ: Oncomirs - microRNAs with a role in cancer. Nature reviews. Cancer 6: 259-269, 2006.

5. Ambros V: The functions of animal microRNAs. Nature 431: 350-355, 2004.

6. Slack FJ and Weidhaas JB: MicroRNA in cancer prognosis. N Engl J Med 359: 2720-2722, 2008.

7. Grimson A, Farh KK, Johnston WK, Garrett-Engele P, Lim LP and Bartel DP: MicroRNA targeting specificity in mammals: determinants beyond seed pairing. Mol Cell 27: 91-105, 2007.

8. Ling B, Wang GX, Long G, Qiu JH and Hu ZL: Tumor suppressor miR-22 suppresses lung cancer cell progression through posttranscriptional regulation of ErbB3. J Cancer Res Clin Oncol 138: 1355-1361, 2012.

9. Pandey DP and Picard D: miR-22 inhibits estrogen signaling by directly targeting the estrogen receptor alpha mRNA. Mol Cell Biol 29: 3783-3790, 2009.

10. Xiong J, Yu D, Wei N, et al: An estrogen receptor alpha suppressor, microRNA-22, is downregulated in estrogen receptor alpha-positive human breast cancer cell lines and clinical samples. FEBS J 277: 1684-1694, 2010.

11. Patel JB, Appaiah HN, Burnett RM, et al: Control of EVI-1 oncogene expression in metastatic breast cancer cells through microRNA miR-22. Oncogene 30: 1290-1301, 2011.

12. Zhang J, Yang Y, Yang T, et al: microRNA-22, downregulated in hepatocellular carcinoma and correlated with prognosis, suppresses cell proliferation and tumourigenicity. Br J Cancer 103: 1215-1220, 2010.

13. Li J, Liang S, Jin H, Xu C, Ma D and Lu X: Tiam1, negatively regulated by miR-22, miR-183 and miR-31, is involved in migration, invasion and viability of ovarian cancer cells. Oncol Rep 27: 1835-1842, 2012.

14. Li J, Liang S, Yu H, Zhang J, Ma D and Lu X: An inhibitory effect of miR-22 on cell migration and invasion in ovarian cancer. Gynecol Oncol 119: 543-548, 2010.

15. Li J, Zhang Y, Zhao J, Kong F and Chen Y: Overexpression of miR-22 reverses paclitaxel-induced chemoresistance through activation of PTEN signaling in p53-mutated colon cancer cells. Mol Cell Biochem 357: 31-38, 2011.

16. Bar N and Dikstein R: miR-22 forms a regulatory loop in PTEN/ AKT pathway and modulates signaling kinetics. PloS One 5: e10859, 2010

17. Yamakuchi M, Yagi S, Ito T and Lowenstein CJ: MicroRNA-22 regulates hypoxia signaling in colon cancer cells. PloS One 6: e20291, 2011.

18. Alvarez-Diaz S, Valle N, Ferrer-Mayorga G, et al: MicroRNA-22 is induced by vitamin D and contributes to its antiproliferative, antimigratory and gene regulatory effects in colon cancer cells. Hum Mol Genet 21: 2157-2165, 2012.

19. Strumane K, Rygiel TP and Collard JG: The Rac activator Tiam1 and Ras-induced oncogenesis. Methods Enzymol 407: 269-281, 2006.

20. Jin H, Li T, Ding Y, et al: Methylation status of T-lymphoma invasion and metastasis 1 promoter and its overexpression in colorectal cancer. Hum Pathol 42: 541-551, 2011.

21. Huang J, Ye X, Guan J, et al: Tiam1 is associated with hepatocellular carcinoma metastasis. Int J Cancer 132: 90-100, 2012. 
22. Yang W, Lv S, Liu X, Liu H and Hu F: Up-regulation of Tiam1 and Racl correlates with poor prognosis in hepatocellular carcinoma. Jpn J Clin Oncol 40: 1053-1059, 2010.

23. Ding Y, Chen B, Wang S, et al: Overexpression of Tiam 1 in hepatocellular carcinomas predicts poor prognosis of HCC patients. Int J Cancer 124: 653-658, 2009.

24. Zhao L, Liu Y, Sun X, He M and Ding Y: Overexpression of $\mathrm{T}$ lymphoma invasion and metastasis 1 predict renal cell carcinoma metastasis and overall patient survival. J Cancer Res Clin Oncol 137: 393-398, 2011.

25. Liu H, Shi G, Liu X, Wu H, Fan Q and Wang X: Overexpression of Tiam1 predicts poor prognosis in patients with esophageal squamous cell carcinoma. Oncol Rep 25: 841-848, 2011.

26. Qi Y, Huang B, Yu L, Wang Q, Lan G and Zhang Q: Prognostic value of Tiam1 and Rac1 overexpression in nasopharyngeal carcinoma. ORL J Otorhinolaryngol Relat Spec 71: 163-171, 2009.

27. Minard ME, Kim LS, Price JE and Gallick GE: The role of the guanine nucleotide exchange factor Tiam1 in cellular migration, invasion, adhesion and tumor progression. Breast Cancer Res Treat 84: 21-32, 2004.

28. Wang HM and Wang J: Expression of Tiam1 in lung cancer and its clinical significance. Asian Pac J Cancer Prev 13: 613-615, 2012.

29. Moriarty CH, Pursell B and Mercurio AM: miR-10b targets Tiam1: implications for Rac activation and carcinoma migration. J Biol Chem 285: 20541-20546, 2010.

30. Cottonham CL, Kaneko S and Xu L: miR-21 and miR-31 converge on TIAM1 to regulate migration and invasion of colon carcinoma cells. J Biol Chem 285: 35293-35302, 2010.
31. Deryugina EI and Quigley JP: Matrix metalloproteinases and tumor metastasis. Cancer Metastasis Rev 25: 9-34, 2006.

32. Patan S: Vasculogenesis and angiogenesis. Cancer Treat Res 117 3-32, 2004

33. Choong ML, Yang HH and McNiece I: MicroRNA expression profiling during human cord blood-derived CD34 cell erythropoiesis. Exp Hematol 35: 551-564, 2007.

34. Xiong J, Du Q and Liang Z: Tumor-suppressive microRNA-22 inhibits the transcription of E-box-containing c-Myc target genes by silencing c-Myc binding protein. Oncogene 29: 4980-4988, 2010.

35. Zhong D, Li Y, Peng Q, et al: Expression of Tiam1 and VEGF-C correlates with lymphangiogenesis in human colorectal carcinoma. Cancer Biol Ther 8: 689-695, 2009.

36. Zhuge $\mathrm{Y}$ and $\mathrm{Xu}$ J: Rac1 mediates type I collagen-dependent MMP-2 activation. role in cell invasion across collagen barrier. J Biol Chem 276: 16248-16256, 2001.

37. Itoh Y, Takamura A, Ito N, et al: Homophilic complex formation of MT1-MMP facilitates proMMP-2 activation on the cell surface and promotes tumor cell invasion. EMBO J 20: 47824793, 2001

38. Shi TY, Cheng X, Yu KD, et al: Functional variants in TNFAIP8 associated with cervical cancer susceptibility and clinical outcomes. Carcinogenesis: January 8, 2013 (Epub ahead of print).

39. Zhang G, Xia S, Tian H, et al: Clinical significance of miR-22 expression in patients with colorectal cancer. Med Oncol 29: 3108-3112, 2012. 